\title{
Influence of Age Specific Parameters on the Thoracic Response under Controlled Belt Loading Conditions
}

\author{
Jacobo Antona-Makoshi ${ }^{1)}$ Yoshihiro Yamamoto $^{2)}$ Ryosuke Kato $^{3)}$ Fusako Sato $^{4)}$ Susumu Ejima ${ }^{5)}$ \\ Yasuhiro Dokko ${ }^{6}$ ) Tsuyoshi Yasuki $^{\text {7) }}$ \\ 1) • 2) - 3) - 4) - 5) Japan Automobile Research Institute \\ 2530 Karima, Tsukuba, Ibaraki, 305-0822, Japan (E-mail: ajacobo@jari.or.jp) \\ 6) - 7) Japan Automobile Manufacturers Association \\ 1-1-30 Shiba Daimon, Minato-ku, Tokyo, 105-0012, Japan
}

Received on June 26, 2014

Presented at the JSAE Annual Congress on May 22, 2014

\begin{abstract}
An anatomically detailed age-specific finite element model of the thoracic region of an average size Japanese elderly male was developed. The model was validated against original series of experimental data both at component and at assembled-structural level. With the validated model, a simulation based age-dependent parametric study was conducted. The results show that rib cortical bone and muscle softening due to ageing affect the structural thoracic response under controlled belt loading conditions. The thoracic model will be implemented into a full scale elderly model and is intended to support the deployment of elderly-specific safety improvement strategies.
\end{abstract}

KEY WORDS: safety, finite element method (FEM), injury mechanism, Thoracic injuries, Elderly [C1]

\section{Introduction}

Elderly accounted for more than half of the 4373 traffic related fatilities in Japan in $2013^{(1)}$. Head, thorax and inner organs were the body regions that comprised the majority of fatal injuries in vehicle occupants. Male elderly drivers were the population group exposed to highest risks. Within this group, thoracic injuries account for as many fatalities as head injuries since $2010^{(2)}$. Increased fragility (break easily) and frailty (do poorly) of the thoracic region due to ageing are two of the factors that may be likely affecting this trend ${ }^{(3)}$. Due to projected ageing population, this situation will worsen in the following years.

To deploy thoracic injury specific safety countermeasures, further invesigation on elderly specific characteristics is necessary. Human Finite Element (FE) age-specific models will be crucial to support such investigations.

The ultimate goal of this work is to reduce thoracic injuries due to traffic crashes, especially in the elderly. The specific aims are two-fold:

- To develop and validate an age-specific thoracic FE model of an average size Japanese elderly motor vehicle occupants.

- To improve the understanding on how age related parameters may affect the thoracic response under controlled belt loading conditions.

\section{Materials}

Computed Tomorgraphy (CT) images of an elderly post mortem human subject (PMHS), and a series of test data obtained from the same specimen (thereafter called baseline specimen) were provided by the University of Virginia Center for Applied Biomechanics laboratory ${ }^{(4)(5)}$. All imaging, testing and handling procedures were reviewed and approved by an independent oversight committee at the University of Virginia.

In addition to the experimental data, a number of software were utilized for image processing (Mimics v11, Materialise, Belgium), geometry generation (Rhinoceros v4, McNeel \& Associates, USA), construction of a mesh (Hypermesh v11, Altair, USA) and FE calculations (ls-dyna mpp971s R5.1.1, LSTC, USA).

\subsection{CT images}

The baseline specimen was a 71 years old male of $161 \mathrm{~cm}$ height and $60 \mathrm{~kg}$ weight. The $\mathrm{CT}$ images were processed to extract the geometry of the spine, the thoracic inner organs, the rib cage including the sternum and the clavicles. The geometry of the rib cage was compared to a sample of post mortem CT data sets obtained from 28 Japanese elderly males at Tsukuba Medical/Autopsy center ${ }^{(6)}$. Table 1 and Fig. 1 show the results of the comparison between the geometry of the baseline specimen used in this study and the Japanese elderly population study ${ }^{(6)}$. It 
was confirmed that the geometry of the rib cage of the baseline specimen fell within 1 standard deviation limits for rib cage bounding box in $\mathrm{x}, \mathrm{y}$ and $\mathrm{z}$ dimensions (Table 1), as well as ribs total lengths and angles with respect to the spine (Fig. 1).

Table 1 Comparison of the baseline specimen ribcage bounding box dimensions to Japanese elderly population study ${ }^{(6)}$.

\begin{tabular}{|c|c|c|}
\hline $\begin{array}{c}\text { Ribcage bounding box } \\
\text { dimensions }\end{array}$ & $\begin{array}{c}65+\text { year old } \\
(\mathrm{n}=28)\end{array}$ & $\begin{array}{c}\text { Baseline } \\
\text { specimen }\end{array}$ \\
\hline $\mathrm{X}(\mathrm{cm})$ & $175(\mathrm{SD} \pm 12.5)$ & 174 \\
\hline $\mathrm{Y}(\mathrm{cm})$ & $268(\mathrm{SD} \pm 21.6)$ & 283 \\
\hline $\mathrm{Z}(\mathrm{cm})$ & $337(\mathrm{SD} \pm 16.3)$ & 352 \\
\hline
\end{tabular}

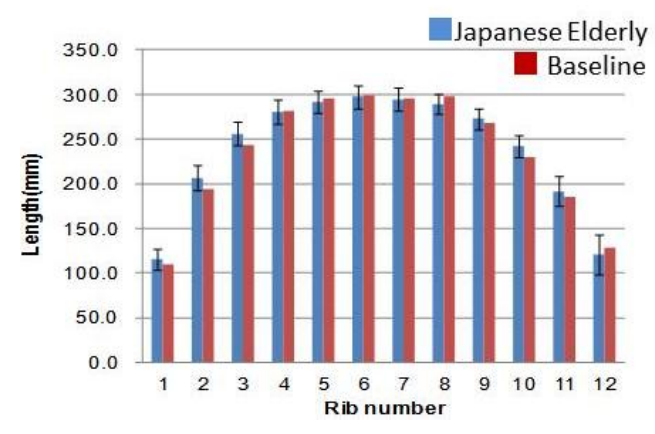

a) Ribs length

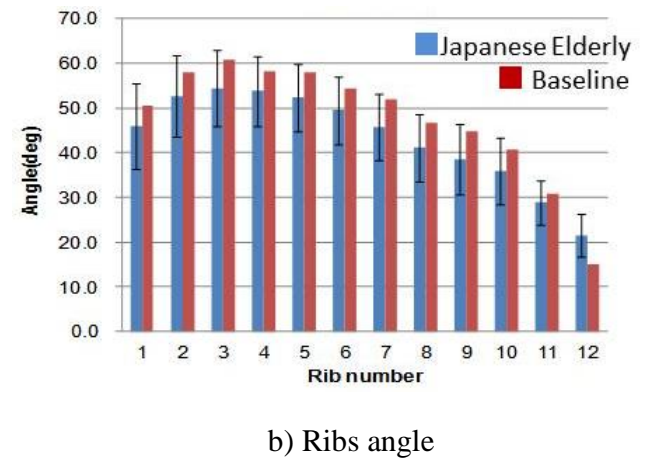

Fig. 1 Comparison of baseline specimen's ribs with average values from a Japanese elderly male population ${ }^{(6)}$.

\subsection{Experimental data}

An original series of sub-injurious level (10\% nominal chest compression) tabletop tests were conducted on the baseline specimen $^{(4)(5)}$. In the experiments, the specimen was positioned supine and rigidly mounted through the spine to a tabletop test fixture. A 5-cm-wide belt, constructed of spectra fiber-reinforced sail cloth to prevent appreciable belt elongation during loading, was positioned across the torso diagonally, passed through D-ring and attached to a trolley driven by a hydraulic ram linked to a universal test machine ${ }^{(4)(5)}$. Ramp and hold anterior thoracic loading was applied at $1.2 \mathrm{~m} / \mathrm{s}$ under three different thoracic tissue conditions in chronological order: intact, denuded (superficial skin, fat and muscles removed) and eviscerated (organs, vasculature and visceral fat removed). During the intact test, one bi-cortical fracture at the $8^{\text {th }}$ left rib (L8) was detected via strain gauges. Besides the bi-cortical fracture detected at the intact case, several minor linear fractures and costal cartilage fractures were reported $^{(5)}$. In the current study, it was assumed that linear fractures did not affect the integrity of the thoracic structure and these fractures were not consider for modeling purposes. The possible influence of the L8 bi-cortical fracture and the costal cartilage fractures are considered in this study for later evaluation of simulation results.

After the tabletop tests, undamaged components of the specimen were harvested and utilized to conduct series of component tests such as rib bending tests ${ }^{(7)}$, intercostal muscle tensile tests ${ }^{(8)}$, and costal cartilage shear tests ${ }^{(9)}$.

\section{Methodology}

\subsection{Development and validation of a thorax FE model of an average size Japanese elderly}

First, the images of the baseline specimen were processed to extract the geometry used to build the baseline model. Then, different components were built and validated separaterly. Thereafter, the validated components were assembled and the thoracic response was validated for three different tissue conditions against the tabletop test experiments ${ }^{(3)(4)}$.

\subsubsection{Model geometry development}

The CT images of the baseline specimen were processed to extract detailed geometry of the thoracic region of the specimen, including ribs, costal cartilage, sternum, clavicles, spine, inner organs (lungs, mediastinum, heart, and a simplified model of the stomach and intenstines) and the outer flesh. Based on this geometry, a mesh was constructed. Bones were modelled as an external layer of shell elements, representing the cortical bone, filled with solid brick elements, representing spongy bone. Boney properties were defined with elasto-plastic materials. Soft tissues and inner organs were modelled with tetrahedral elements. Viscoelastic material properties of different charachteristics, depending on available experimental data in literature were utilized. Lung and mediastinum models share nodes with the internal wall of the rib cage. The rest of the attachments between and within organs and surrounding structures were defined based on anatomy literature and upon consultation with forensic physicians. Balance between model geometric accuracy and computational time requirements was achieved by establishing a 
target of maximum calculation timestep of 0.3 micro seconds. Fig. 2 shows images of the rib cage, the inner organs and the outer flesh of the developed model.

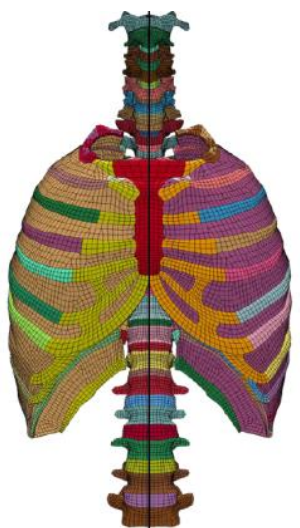

a) Frontal view of ribcage and spine

Outer Flesh

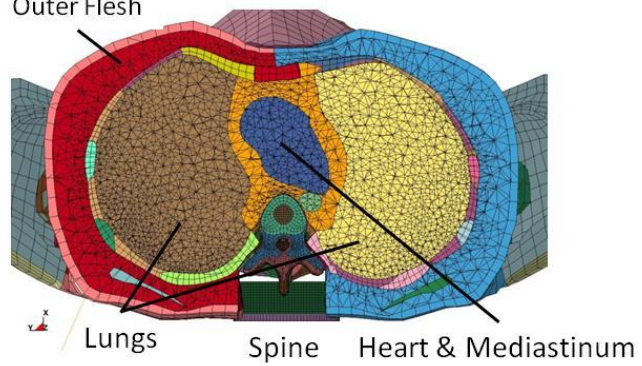

c) Thoracic transversal view of inner organs and flesh

Fig. 2 Images of the developed model

\subsubsection{Thoracic component models and validation}

Ribs, costal cartilage and intercostal muscles were validated against component test data obtained from the baseline specimen $^{(7)-(9)}$. Inner organs materials were defined and validated based on data from the literature.

\section{Ribs}

A modeling methodology proposed by Kato et al. ${ }^{(10)}$ was utilized to build and validate the ribs models. The methodology consists on extracting the variable cortical thickness from the CT images and automatically implement them into the rib models across each section and along each rib. Based on the developed geometry, a rib bending test fixture model was developed and bending tests were simulated. A reverse engineering loop was applied to the simulation by varying rib cortical Young's modulus, yield stress, and ultimate strain values within ranges available in literature until optimum combination of parameters was found. Such modelling technique was successful in reproducing with accuracy force displacement behaviour from a number of rib bending tests ${ }^{(10)}$.
In the current work, the modeling approach by Kato et al. ${ }^{(10)}$ was applied to develop all the ribs from the baseline model's CT and to validate 9 of them against bending tests with ribs harvested from the baseline specimen. Fig. 3 shows an example of the comparison of the simulation and the experimental force displacement curves resulting from the $6^{\text {th }}$ left rib of the baseline specimen.

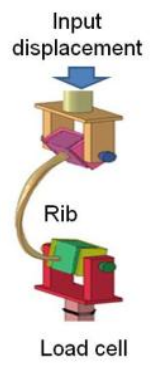

a) Simulation setup

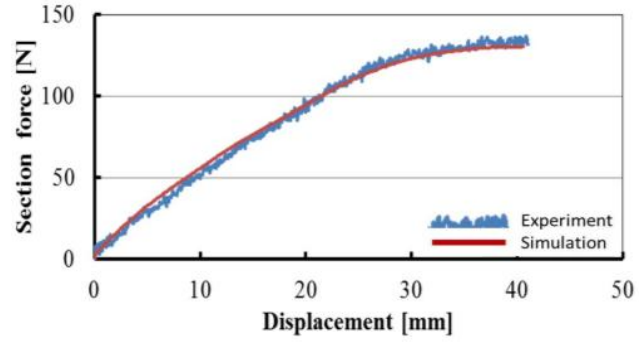

b) Force - Displacement experiment and simulation results
Fig. 3 Rib bending test model simulation setup and validation

\section{Inner organs}

The liver was modeled as an Ogden rubber material with hyper-elastic and rate-dependent characteristics. The model was validated against original series of porcine exsanguinated livers under quasi-static and dynamic compression experiments. The applicability of the validated liver FE model was later evaluated against series of high-rate compression tests with porcine perfused livers to model nearly in vivo conditions. More details of the liver model, the experiments and the validation process can be found in Sato et al. ${ }^{(11)}$. The properties of the lungs and the mediastinum were defined based on a material model proposed by MendozaVazquez et al. ${ }^{(12)}$ which was validated with drop tests to perfused porcine lungs ${ }^{(13)}$.

\section{Costal cartilage and intercostal muscles}

Costal cartilage was modeled as an elasto plastic material with properties by Yamada et al. ${ }^{(14)}$ for 70 's year old population. The cartilage model was validated against shear experiments also conducted on the baseline specimen ${ }^{(9)}$. The intercostal muscles were modeled as a viscoelastic material and validated against intercostal muscle tensile tests also conducted on samples from the baseline specimen ${ }^{(8)}$.

\section{Outer Flesh}

A simplified model of the outer thoracic flesh, including fat and thoraco-abdominal muscles was built by filling the volume occupied by these structures with combined brick and tetrahedral elements. A viscoelastic material model was defined based on 
cadaver muscle coupon tests in compression ${ }^{(15)}$ and in tensile according to 70's year old group muscle tissue properties ${ }^{(14)}$.

\subsubsection{Simulation of Tabletop tests: Thoracic structural} stiffness validation and predictability of fractures

The validated components were assembled and the structural response of the rib cage was validated by simulating the tabletop tests $^{(4)(5)}$. A geometrically accurate FE model of the spine contstraint system and the load cells array system were developed from the CT images of the specimen, which were taken from the subject with the spinal support bar in the "as tested" position. This provided a quantitative 3-D assessment of the spine posture as well as detailed measurements of all installed hardware relative to an anatomical reference frame ${ }^{(4)}$. The model of the table frame and the carriage used in the experiments was developed upon consultation with the authors of the experiments ${ }^{(4)}$ (See Fig. 4). This process allowed to replicate with accuracy the positioning of the thoracic model and the spinal fixture by importing the VICON data from the experiments with respect to the table frame.

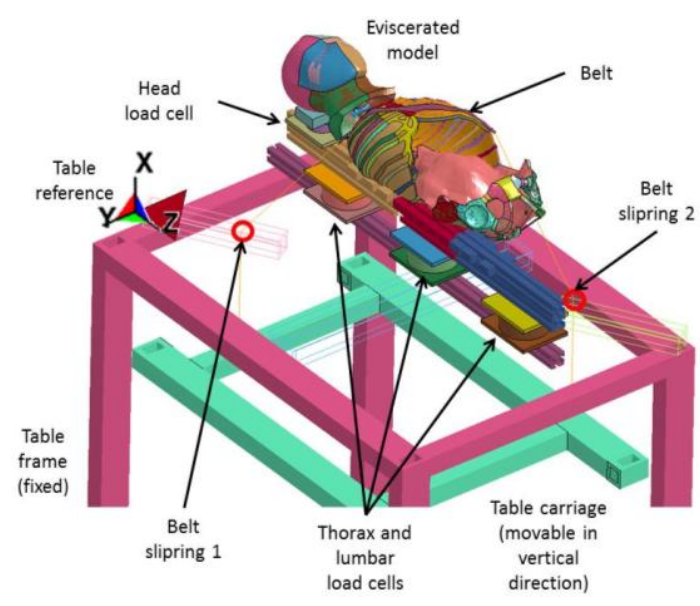

Fig. 4 Tabletop test simulation setup.

Three different versions of the thoracic model were built to replicate intact, denuded and eviscerated tissue conditions. The VICON data on the belt and the pulleys were also utilized to build a model of the spectra fiber-reinforced sail cloth belt ${ }^{(5)}$. To simulate the experiments, the carriage displacement curve measured at each of the experiments ${ }^{(5)}$ was prescribed to the carriage in the FE model. $100 \mathrm{~ms}$ preloading was applied in the simulations, followed by $35 \mathrm{~ms}$ of dynamic loading up to approximately $30 \mathrm{~mm}$ table displacement. Fig. 5 shows the models used and the results of comparing the simulations with the experiments for each of the tissue conditions. Force-displacement curves show belt force as measured at the right shoulder side ${ }^{(3)}$ and the table carriage displacement applied to the belt ends both in the experiments ${ }^{(5)}$ and in the simulations.

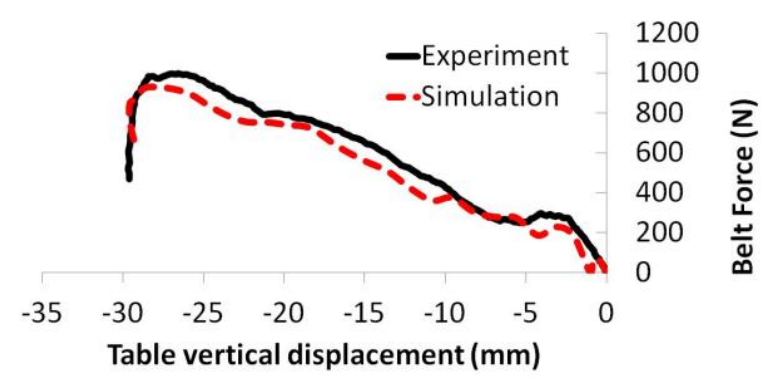

a) Intact condition

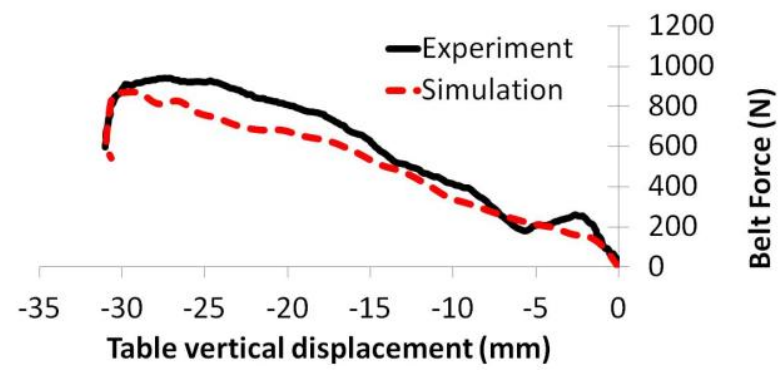

b) Denuded condition

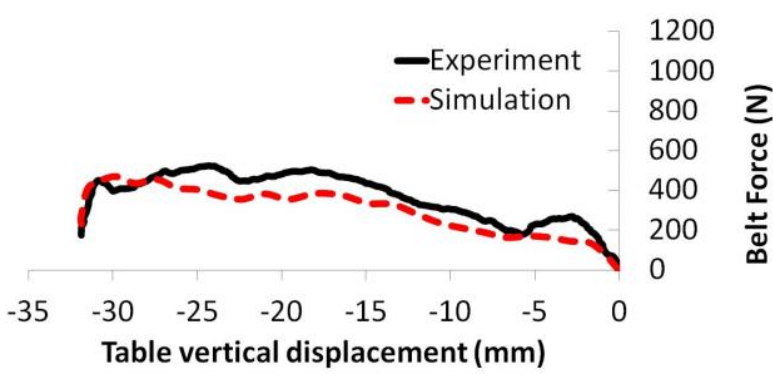

c) Eviscerated condition

Fig. 5 Assembled model validation

\section{Predictability of rib fracture}

According to the experimental study ${ }^{(5)}$ one bi-cortical fracture occurred at the eighth left rib (L8) in the intact case experiment. The strain profile captured via strain gauges placed on $4^{\text {th }}, 6^{\text {th }}, 8^{\text {th }}$ and $10^{\text {th }}$ ribs in the experiment is shown in Fig 6 .

The peak maximum principal strains obtained from the corresponding intact case simulation at the same rib levels are also presented. These peaks occured on the lateral part of the ribs at the time of maximum thoracic deflection.

The strain profiles on the left side, as well as the differences between right and left sides were similar in the experiment and in the simulation. A maximum strain value of $0.75 \%$ was measured at L8 in the experiments, while in the simulation, a peak maximum principal strain (MPS) of $0.56 \%$ occured at the same rib. Both the experimental and the simulation values are higher 
than the thresholds above which cortical fracture may occur according to Kemper et al. ${ }^{(16)}$.
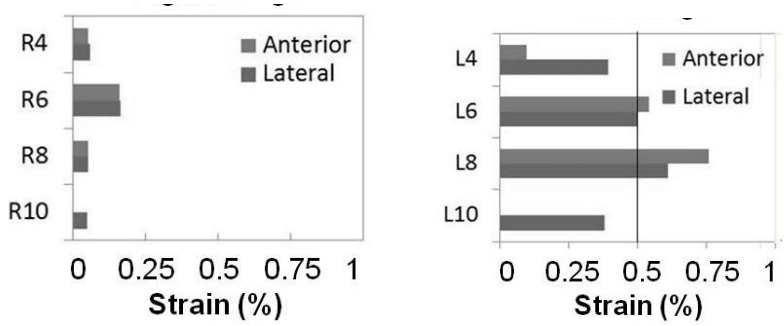

a) Right ribcage test strains

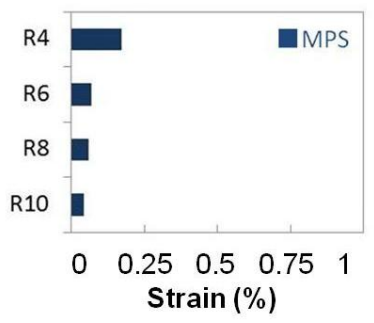

c) Right ribcage MPS from simulation

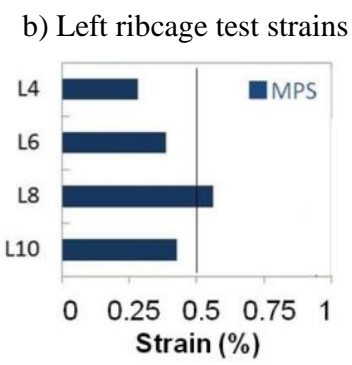

d) Left ribcage MPS from simulation

Fig. 6 Comparison of maximum strains measured at the experiment with intact conditions and MPS from the corresponding simulations.

\section{Predictability of costal cartilage fractures}

Costochondral junction fractures at R1 and L1 level as well as sternochondral junction fracture at the low right side of the sternum were found during necropsy ${ }^{(4)}$. Hence, it was uncertain when such injuries occurred. In the intact case simulation, fractures occurred at the costochondral junction at R1 and L1 level as well as at the low right side of the sternum. Such fractures occurred in the simulations at maximum sternal deflection under an assymetric loading mechanism, as illustrated in Fig. 7.

\subsection{Age dependent parametric study}

A four-parameter two-level full factorial simulation parametric study was conducted with the validated model under tabletop test intact condition. The output variable chosen was thoracic effective stiffness defined as the inclination of the slope of the force-displacement curve (See Fig. 5) previously described. Elderly and Adult levels were defined for each paramter. To set such levels, it was assumed that the baseline specimen was representative of elderly conditions and each parameter was scaled to adult conditions as follows.

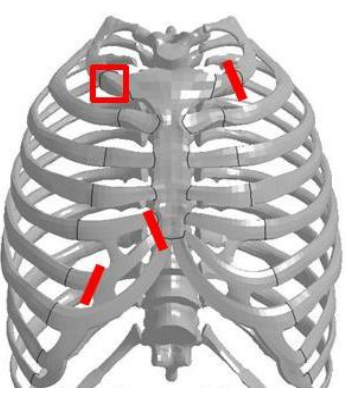

a) Experimental Costal cartilage fractures $^{(5)}$

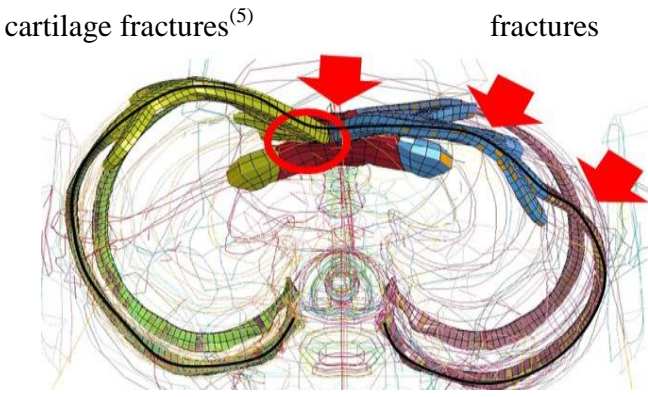

c) Loading mechanism producing costal cartilage fractures

Fig. 7 Predictability of costal cartilage fractures

\subsubsection{Rib cortical bone thickness}

Rib cortical bone thickness decreases with age ${ }^{(17)}$. Based on this, scaling factors for rib cortical bone thickness as a function of age were proposed in a FE modelling study ${ }^{(18)}$. To define the scaling factors, a relationship between cortical section and age was utilized $^{(17)}$. In the current study, the variable thickness of all ribs was obtained from the $\mathrm{CT}$ images of the baseline specimen and implemented into the elderly model. Then, a factor of 1.3 to the rib cortical thickness values from Ito et al. ${ }^{(18)}$, in order to scale the baseline elderly (70 years old) rib cortical bone thicknesses to adult (35 years old).

\subsubsection{Rib cortical bone material properties}

Rib cortical ultimate tensile strain decreases with age $^{(14)(16)(22)}$. In the Ito et al. study ${ }^{(18)}$ a method to scale cortical bone properties from adult to elderly was proposed. Such method applies a reduction of elastic modulus and plastic stress to scale from adults to elderly. Such scaling was supported by a study showing decreasing ultimate stress with age of femur cortical bone $\mathrm{e}^{(18)}$ and is consistent with other studies on femora and tibiae $^{(14)(20)}$. In the current study, the same scaling approach and factors was applied to derive adult properties from the properties defined for the baseline elderly model as presented in Fig 8.a

\subsubsection{Costal cartilage material properties}

Age dependent tensile stress-strain properties of costal cartilage of 28 cadavers from ages ranging from 20 to 69 years 
old have been reported by Yamada et al. ${ }^{(13)}$ and utilized in an FE study $^{(18)}$. Based on these data, properties for adults and elderly were applied to the model as shown in Fig 8.b.

\subsubsection{Thoracic flesh material properties}

Age-dependent tensile propeties of muscles have been reported ${ }^{(13)}$. Assuming that the changes of muscle properties with age are proportional in compression and in tension, the outer flesh curves previously defined for elderly were scaled up to adult properties according to Yamada's tensile test data as shown in Fig 8.c.

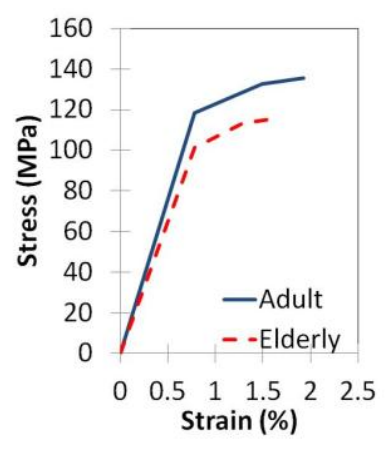

a) Rib cortical bone

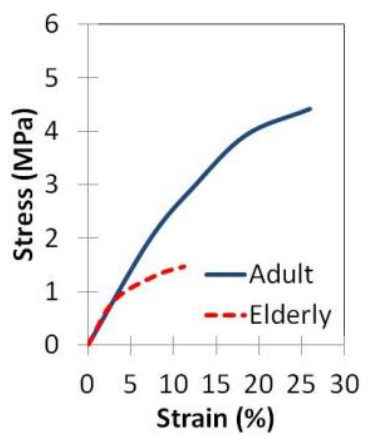

b) Costal cartilage

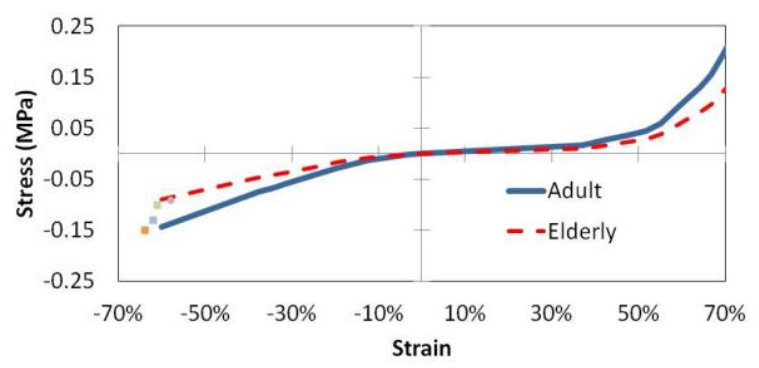

c) Thoracic flesh

Fig. 8 Age-dependent thoracic material properties

\section{Results}

Table 2 shows the parametric study design matrix and the resulting of effective stiffness for each case. Fig 9 shows forcedisplacement curves of the experiment, the most compliant simulation case (case 1; baseline with all Elderly-compliant properties), and the stiffest simulation case (case 16; all Adultstiff properties). All other simulated cases showed intermediate response according to values presented in Effective Stiffness column in Table 2.

When adult properties were implemented, the model responded to pre-defined belt displacements with a total increase of effective stiffness of $28.5 \%$. The main effect of age dependent outer flesh properties accounted for a $15.9 \%$ of such increase. The main effect of rib cortical bone changes contributed with a $7.6 \%$ of the total increase, equally divided between thickness (3.8\%) and material properties $(3.8 \%)$. Finally, the main effect of agedependent properties of the costal cartilage accounted for a $5 \%$ of the total stiffening. It is however worthy to mention here that, although the influence on stiffness was the smallest, cartilage fractures occurred only in the simulated cases in which elderly properties were implemented (cases 1-4 and 9-12).

Table 2 Parametric study design matrix and results

\begin{tabular}{|c|c|c|c|c|c|}
\hline Case & $\begin{array}{c}\text { Rib } \\
\text { Cortical } \\
\text { Thickness }\end{array}$ & $\begin{array}{c}\text { Rib } \\
\text { Propertical }\end{array}$ & $\begin{array}{c}\text { Costal } \\
\text { Cartilage } \\
\text { Properties }\end{array}$ & $\begin{array}{c}\text { Outer } \\
\text { Plesh }\end{array}$ & $\begin{array}{c}\text { Effective } \\
\text { Stiffness } \\
\text { (N/cm) }\end{array}$ \\
\hline 1 & Elderly & Elderly & Elderly & Elderly & 315 \\
\hline 2 & Adult & Elderly & Elderly & Elderly & 334 \\
\hline 3 & Elderly & Adult & Elderly & Elderly & 333 \\
\hline 4 & Adult & Adult & Elderly & Elderly & 341 \\
\hline 5 & Elderly & Elderly & Adult & Elderly & 336 \\
\hline 6 & Adult & Elderly & Adult & Elderly & 346 \\
\hline 7 & Elderly & Adult & Adult & Elderly & 346 \\
\hline 8 & Adult & Adult & Adult & Elderly & 354 \\
\hline 9 & Elderly & Elderly & Elderly & Adult & 373 \\
\hline 10 & Adult & Elderly & Elderly & Adult & 379 \\
\hline 11 & Elderly & Adult & Elderly & Adult & 380 \\
\hline 12 & Adult & Adult & Elderly & Adult & 389 \\
\hline 13 & Elderly & Elderly & Adult & Adult & 386 \\
\hline 14 & Adult & Elderly & Adult & Adult & 394 \\
\hline 15 & Elderly & Adult & Adult & Adult & 395 \\
\hline 16 & Adult & Adult & Adult & Adult & 405 \\
\hline
\end{tabular}

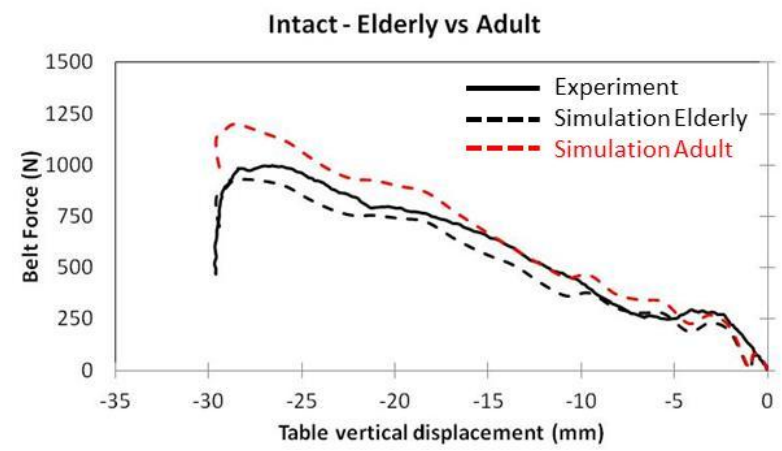

Fig .9 Force-displacement experimental curve, simulated curve obtained for case 1 (Elderly) and case 16 (Adult).

\section{Discussion}

The structural response of the developed elderly thorax model has been validated against experiments conducted on the baseline specimen under Intact, Denuded and Eviscerated tissue conditions (4)(5). When the thoracic outer flesh was removed, the resulting 
effective stiffness decreased a $13 \%$ with respect to the Intact case. When the inner organs were removed, the overall stiffness was about half of that in the Intact case. This large effect of removing the inner organs observed in our model and corresponding experiment is consistent to comparable experimental results by Kent et al. ${ }^{(24)}$.

$\mathrm{Rib}$ fractures were not expected to occur in the tabletop tests. However, L8 fractured at the intact experiment at a low level of strain $(0.75 \%)$. This level is above the threshold of $0.5 \%$ for rib cortical bone above which fracture may occur ${ }^{(16)}$. When the same experimental case was simulated, a strain of $0.56 \%$ was obtained. This value was slightly smaller than the one measured at the experiment but still above the $0.5 \%$ threshold $^{(16)}$. Hence, according to this threshold, the model developed produced strains in the same approximate location and order of magnitude as the experiments, which indicates the model's potential to predict rib fractures.

Ultimate strain of cortical bone decreases as we age ${ }^{(14)(16)(22)}$. In this study, the age-scaling method by Ito et al. ${ }^{(17)}$ was adopted. Such study, supported by Dokko et al. ${ }^{(23)}$ and Burstein et al. ${ }^{(19)}$, hypothesizes that the decrease of ultimate strain is accompanied by a decrease in tandem of elastic modulus and ultimate stress. When implementing such changes in the parameter study, it was confirmed that the age-dependent parameter affected a $3.8 \%$ to the thoracic effective stiffness. This estimation is consistent with the $6 \%$ obtained by Kent et al. ${ }^{(22)}$ in a comparable numerical study.

Thoracic flesh, fat, and muscles may play an important role in protecting the thorax. Some studies have suggested that obese occupants may be subjected to lower thoraco-abdominal injury risks $^{(26)}$. However, no studies on the possible effect of aged outer flesh on possible injury outcome has been found. By the age of 30 , a gradual loss of muscle mass called sarcopenia, begins to occur. Sarcopenia is the degenerative loss of skeletal mass, quality, and strength associated with aging. Muscle strength has usually decreased by about $50 \%$ by the age of 80 years $^{(25)}$. This decrease is consistent with age dependent material properties reported by Yamada et al. ${ }^{(14)}$ and was implemented in the model. By considering such differences of mechanical properties without varying the geometry of the flesh, we could verify that the flesh did play an important role on the structural response of the thorax and should be considered in future age-dependent studies.

\section{Limitations and future work}

Most of the validation presented has been conducted using experimental data from a single elderly PMHS. Although the specimen's ribcage geometry falls within the range of the average elderly Japanese, uncertainties remain on how representative this specimen was with respect to a wider population. In addition, the tabletop tests were conducted prior to harvesting parts for component tests, which may have affected the integrity of some of the components used.

Contrary to the hypothesis used in this study to scale rib cortical bone properties by Ito et al. ${ }^{(18)}$, an alternative scaling methodology in which elastic modulus and ultimate stress are suggested to increase with age has been proposed by Iwamoto et al. ${ }^{(19)}$ based on a series of rib cortical bone coupon tensile tests from 6 PMHS of different ages ${ }^{(16)}$. Adopting such an alternative approach for age scaling of cortical bone properties would affect the tendency of the results presebted in this work. This controversy calls for further experimental work to clarify how rib cortical material properties change with age.

Rib cage geometry may change with age ${ }^{(22)}$. Under tabletop test conditions, geometry changes such as rib orientation have been suggested to affect thoracic structural stiffness ${ }^{(22)}$. In our study, geometrical changes have not been considered. As more age specific population based geometrical data are generated $^{(21)}$, age dependent geometry studies will become possible.

Finally, the severity of the loads applied in this study is lower than injurious car crash conditions. Hence, the conclusions obtained from this work may not be directly applicable to full scale conditions. The thoracic model developed and presented in this study demands further validation at more severe loading conditions.

\section{Conclusions}

An FE model of the thoracic region has been developed from CT images of an elderly PMHS which body weight and height and rib cage geometry fall within average Japanese elderly male characteristics. The model has been validated both at component and assembled level against original series of experimental data from the same specimen. In the developed model, consistently with the experiments ${ }^{(4)(5)}$, the major contributor to structural response under controlled belt loading was the ribcage, followed by the inner organs.

When adult properties were considered instead of the original elderly properties, the model responded with a $28.5 \%$ increase in structural stiffness. In descending order, outer flesh, rib cortical thickness and properties, and costal cartilage age-dependent changes contributed to such increase.

Despite working at non-injurious levels, the thoracic model developed presented sensitivity to age dependent characteristics, showing its potential to discriminate age specific characteristics 
potentially relevant for thoracic injury prevention. The model has been implemented into a full body scale model and it is undergoing validation at injurious level conditions ${ }^{(27)}$. The full scale model will be used to research and develop elderly specific safety countermeasures.

\section{REFERENCES}

(1) Japanese Police: Annual report on road traffic fatal accidents and traffic law enforcement violations, (2013). Available at: https://www.npa.go.jp/toukei/index.htm\#koutsuu. Accessed: June 25, 2014. (In Japanese)

(2) Yaguchi M et al: Traffic accident analysis towards the development of and advanced frontal crash test dummy indispensable for further improving vehicle occupant protection performance. Proceedings of the 22nd ESV Conference (2011).

(3) Wang S: Population variability: Influence of Crash Injury Outcomes. JSAE Forum Text, No.13FORUM-18, pp. 1-16 (2013).

(4) Salzar R and Subit D: Development of a Transfer Function for the Evaluation of the Elderly Thoracic Response, JSAE

Proceedings No. 70-14, pp. 7-12 (2014)

(5) Subit D and Salzar R: Rib cage kinematics under belt loading in intact, denuded and eviscerated conditions, JSAE Proceedings No. 70-14, pp. 1-6 (2014)

(6) Ejima S et al: Morphology analysis with Autopsy imaging for characterizing the human rib cages geometry, Proceedings of 19th Japan Society for Computational Engineering and Science (2014) (In Japanese).

(7) Perz R et al: Evaluation of the Geometrical Properties Distribution Along the Human Ribs Using Different X-Ray Imaging Methods, IRCOBI conference (2013).

(8) Hamzah M et al: An Inverse Finite Element Approach for Estimating the Fiber Orientations in Intercostal Muscles, IRCOBI conference (2013)

(9) Subit D and Forman J: Shear loading of costal cartilage. Arxiv 2014. Available at: http://arxiv.org/abs/1405.2308 . Accessed: June 25, 2014.

(10) Kato et al: A methodology to develop rib finite element models that account for cortical bone thickness, JSAE

Proceedings No. 39-14, pp. 1-6 (2014) (In Japanese)

(11) Sato F et al: Hyper-viscoelastic Response of Perfused Liver under Dynamic Compression and Estimation of Tissue Strain Thresholds with a Liver Finite Element Model, IRCOBI conference (2013).

(12) Mendoza-Vazquez M et al: Human rib response to different restraint systems in frontal impacts: a study using a human body model. International Journal of Crashworthiness, Vol.18, No. 5, pp 516-529 (2013)
(13) Hayamizu N et al: Measurement of impact response of pig lung. Japan Society of Mechanical Engineering (JSME) conference (2003) (In Japanese).

(14) Yamada H and Evans FG: Strength of biological materials. Williams \& Wilkins (1970)

(15) McElhaney J et al : Handbook of Human Tolerance, printed by JARI, Japan (1976)

(16) Kemper A et al: Material Properties of Human Rib Cortical Bone from Dynamic Tension Coupon Testing, Stapp Car Crash Journal, Vol. 49 (2005)

(17) Stein ID and Granik G: Rib structure and bending strength: an autopsy study. Calcified tissue research, Vol. 20, No.1, pp 6173 (1976).

(18) Ito O, Dokko Y, and Ohashi K: Development of adult and elderly FE thorax skeletal models. SAE Technical Paper, 200901-0381 (2009)

(19) Iwamoto M et al: Development of a finite element model of a 5th percentile female with multiple muscles and its application to investigation on impact responses of elderly females. Proceedings of the 23rd ESV conference (2013)

(20) Burstein AH and Martens DT: Aging of Bone Tissue: Mechanical Properties, The Jouranl of Bone and Joint Surgergy, Vol 58, pp.82-86 (1976).

(21) Holcombe S et al: Age-based Predictive Model of the Pediatric Rib cage, International Journal of Automotive Engineering, Vol.5, No.1, pp.15-22, (2014)

(22) Kent R et al: Structural and material changes in the aging thorax and their role in crash protection for older occupants.

STAPP Car Crash Conference, Vol. 49, pp. 231-249 (2005)

(23) Dokko Y et al: Development of the Human Lower Limb FE Models considering Aging Effects. SAE Technical Paper 200901-0396 (2009)

(24) Kent R et al: Frontal Thoracic Response to Dynamic Loading the Role of Superficial Tissues, Viscera and the Rib cage. Proceedings of the IRCOBI Conference (2005)

(25) Marieb E: Human anatomy and physiology. Pearson International Edition (2010)

(26) Wang SC et al: Increased depth of subcutaneous fat is protective against abdominal injuries in motor vehicle collisions. Annu. Proc. Assoc. Adv. Automot. Med. Vol. 47 (2003),

(27) Yamamoto Y et al: Kinematics and thoracic deformation of an elderly size human FE model in front impact sled analysis. JSAE Proceedings No. 39-14, pp. 21-26 (2014) (In Japanese) 